\title{
Rich Dynamics of a Brucellosis Model with Transport
}

\author{
Juan Liang $\mathbb{D}^{1,2}$ Zhirong Zhao, ${ }^{1}$ and ${\mathrm{Can} \mathrm{Li}^{1}}^{1}$ \\ ${ }^{1}$ Department of Science, Taiyuan Institute of Technology, Taiyuan, Shanxi 030008, China \\ ${ }^{2}$ Data Science and Technology, North University of China, Taiyuan, Shanxi 030051, China \\ Correspondence should be addressed to Juan Liang; liangjuan76@126.com
}

Received 11 June 2020; Accepted 14 July 2020; Published 6 August 2020

Academic Editor: Xinzhu Meng

Copyright $\odot 2020$ Juan Liang et al. This is an open access article distributed under the Creative Commons Attribution License, which permits unrestricted use, distribution, and reproduction in any medium, provided the original work is properly cited.

Brucellosis is one of the major infectious diseases in China. In this study, we consider an SI model of animal brucellosis with transport. The basic reproduction number $\mathscr{R}_{0}$ is obtained, and the stable state of the equilibria is analyzed. Numerical simulation shows that different initial values have a great influence on results of the model. In addition, the sensitivity analysis of $\mathscr{R}_{0}$ with respect to different parameters is analyzed. The results reveal that the transport has dual effects. Specifically, transport can lead to increase in the number of infected animals; besides, transport can also reduce the number of infected animals in a certain range. The analysis shows that the number of infected animals can be controlled if animals are transported reasonably.

\section{Introduction}

Brucellosis is a zoonosis. It is one of the animal diseases, especially domesticated livestock. Brucellosis mainly attacks sheep, cattle, horses, dogs, pigs, humans, and so on. This epidemic is highly infectious and can be transmitted directly or indirectly through brucella carriers. Humans and animals have certain immunity after suffering from the disease, but they can be infected again. Its incidence is higher in spring and summer. Humans are generally susceptible to the disease and can acquire certain immunity. There is cross immunity among different Brucella species, and the reinfection rate is $2-7$. Some scholars have studied the infectious diseases and obtained some results [1-8].

Many articles have studied brucellosis through dynamic models [9-12]. Hou et al. [9] proposed a model with general incidences and analyzed the dynamics of this model. Sun and Zhang [11] gave a brucellosis model which incorporated proportional birth and studied the global stability. Yang et al. [13] proposed to combine age structured brucellosis disease model with spatial diffusion infection firstly $\left(R_{0}\right.$ was obtained by mathematical analysis), analyzed the sensitivity to parameters, and gave the prevention and control measures of the disease. Yang et al. [14] studied a nonlinear model including spatial and seasonal variations to study the transmission dynamics of brucellosis and proved the importance of spatial and seasonal heterogeneity in disease control. The mathematical model of sheep brucellosis and the effect of slaughtering policy have also been studied [15].

The Chinese government has taken many measures to prevent diseases: vaccination, disinfection and elimination, and so on. If the disease is found, culling measure is taken immediately. However, in fact, some breeders do not take these measures in order to reduce economic losses, which can cause the spread of disease; besides, because the disease has a latent period, breeders do not discover brucellosis in time. In these cases, they transport animals which include the infected.

There has been some studies on the impact of transport on disease transmission [16-18]. A delay periodic patch model with transmission related infection was studied and the authors discussed the difference between the periodic model and the corresponding autonomous model [19]. Liu and Stechlinski [20] proposed a periodic SIS epidemic model with time delay and transport related infection in patchy environment. They discussed the dependence of $R_{0}$ on transport related infection parameters and fluctuation amplitude. This paper studies the disease transmission of the remaining animals after the animals are transported away, which will be helpful for disease prevention and control. 
In this paper, we study an SI model of animal brucellosis with transport. We show that transport has dual effects. In Section 2, we study the existence of the equilibrium. In Section 3, we study the dynamic behavior of the equilibrium. In Section 4, the numerical simulation is given. Finally, some conclusion are summarized and discussed.

\section{Equilibria}

We study a two-dimensional model: densities of the susceptible $(S)$ and densities of the infected $(I)$, in this paper. It is assumed that the transport rate $B$ is a nonzero constant when the number of infected is less than a certain value; when the number of infected is greater than a certain value, $B$ equals 0 . The detailed relation between susceptible and infected is given in Figure 1. Then, the model is expressed in the following form:

$$
\left\{\begin{array}{l}
\dot{S}=A-B C-\beta S I-d S \\
\dot{I}=-B(1-C)+\beta S I-d I-u I
\end{array}\right.
$$

where

$$
\begin{cases}B \neq 0, & \text { if } I \leq I_{0} \\ B=0, & \text { if } I>I_{0} .\end{cases}
$$

We assume that all parameters are nonnegative in this model.

$A$ is the recruitment rate of the animal populations, $\beta$ is the infection coefficient, $B$ is transport proportion, $0<C<1$ is the rate of susceptible in this transport, $d$ is the natural death rate of the animal populations, and $\mu$ is the diseaserelated death rate.

When $I \leq I_{0}$, the steady state of (1) can be written as follows:

$$
\left\{\begin{array}{l}
A-B C-\beta S I-d S=0, \\
-B(1-C)+\beta S I-d I-u I=0 .
\end{array}\right.
$$

When $I>I_{0}$, the steady state of (1) can be written as follows:

$$
\left\{\begin{array}{l}
A-\beta S I-d S=0 \\
\beta S I-d I-u I=0
\end{array}\right.
$$

(1) has no disease-free equilibrium.

Based on Ref. [21], we can obtain the basic reproduction number $\mathscr{R}_{0}$ :

$$
\begin{array}{r}
\mathscr{F}=[\beta S I], \\
v=[(d+u) I], \\
F=\beta S=\frac{\beta A}{d}, \\
V=d+u, \\
\mathscr{R}_{0}=\rho\left(F V^{-1}\right)=\frac{\beta A}{d(d+u)} .
\end{array}
$$

System (4) has a positive equilibrium $E^{*}=\left(S^{*}, I^{*}\right)=$ $\left((d+u) / \beta,\left(d\left(\mathscr{R}_{0}-1\right)\right) / \beta\right)$.

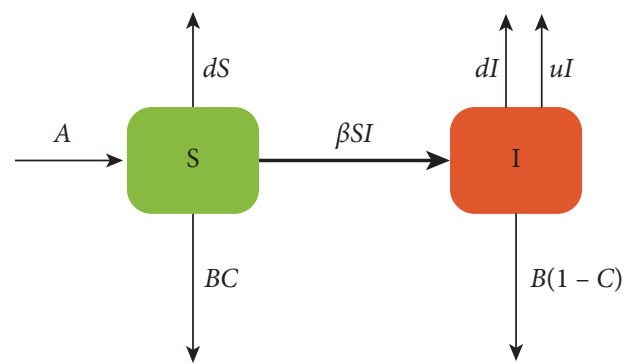

Figure 1: Flow diagram of disease transmission. Here, $S$ denotes susceptible, $I$ denotes infected, $B$ is transport proportion, and $C$ is the proportion of susceptible in the transport.

Evidently, we have

$$
\mathscr{R}_{0}>\frac{\beta I_{0}}{d}+1 .
$$

Next, we calculate the positive solution of system (3). Through the first equation of system (3), we obtain $S=(A-B C) / \beta I+d$. Then, we substitute it into the other equation of (3), so we have

$$
\beta(d+\mu) I^{2}+\left(\mu d+d^{2}+B \beta-A \beta\right) I+B d(1-C)=0 .
$$

Assume $b=\mu d+d^{2}+B \beta-A \beta$. Note that $A-B C>0$.

If $b \geq 0$, it is easy to know that there is no positive equilibrium; then, we consider $b<0$, where

$$
b=\mu d+d^{2}+B \beta-A \beta=\left(-\mathscr{R} d(d+u)+u d+d^{2}+B \beta\right) .
$$

Consequently, we have the following expression:

$$
\begin{aligned}
\Delta= & \left(-\left(-\mathscr{R} d(d+u)+u d+d^{2}+B \beta\right) d(d+u)+u d+d^{2}+B \beta\right)^{2} \\
& -4 \beta(d+\mu)(1-C) B d .
\end{aligned}
$$

$\Delta \geq 0$ is equivalent to

$$
\mathscr{R}_{0} \leq 1+\frac{B \beta}{d(d+u)}-\frac{2 \sqrt{\beta(d+\mu)(1-C) B d}}{d(d+\mu)},
$$

$$
\mathscr{R}_{0} \geq 1+\frac{B \beta}{d(d+u)}+\frac{2 \sqrt{\beta(d+\mu)(1-C) B d}}{d(d+\mu)} \triangleq p_{0} .
$$

$b<0$ is equivalent to

$$
\mathscr{R}_{0} \geq 1+\frac{B \beta}{d(d+u)} .
$$

$b<0$ and $\Delta \geq 0$ if and only if (10) holds. If (10) holds, there are two solutions of (3):

$$
\begin{aligned}
& I_{1}=\frac{-b-\sqrt{\Delta}}{2 \beta(d+\mu)}, \\
& I_{2}=\frac{-b+\sqrt{\Delta}}{2 \beta(d+\mu)} .
\end{aligned}
$$

If the condition $I_{1} \leq I_{0}$ holds, then we have 


$$
-b-\sqrt{\Delta}<2 \beta(d+\mu) I_{0} .
$$

This is equivalent to

$$
\left\{\begin{array}{l}
b+2 \beta(d+\mu) I_{0} \leq 0, \\
\left(b+2 \beta(d+\mu) I_{0}\right)^{2} \leq \Delta,
\end{array}\right.
$$

or

$$
b+2 \beta(d+\mu) I_{0} \geq 0 .
$$

By calculating, (14) is equivalent to

$$
\left\{\begin{array}{l}
\mathscr{R}_{0} \geq 1+\frac{B \beta}{d(d+u)}+\frac{2 \beta I_{0}}{d} \stackrel{\Delta}{=} p_{1}, \\
\mathscr{R}_{0} \geq 1+\frac{B \beta}{d(d+u)}+\frac{\beta I_{0}}{d}+\frac{(1-C) B}{(d+u) I_{0}} \triangleq p_{2} .
\end{array}\right.
$$

(15) is equivalent to

$$
\mathscr{R}_{0} \leq p_{1} .
$$

As a conclusion, when $\mathscr{R}_{0} \geq \max \left(p_{1}, p_{2}\right)$ or $\mathscr{R}_{0} \leq p_{1}$, $I_{1}<I_{0}$ holds.

If $I_{2}<I_{0}$ holds, we have

$$
-b+\sqrt{\Delta} \leq 2 \beta(d+\mu) I_{0} .
$$

It is equivalent to an inequality as follows:

$$
\left\{\begin{array}{l}
b+2 \beta(d+\mu) I_{0} \geq 0, \\
\left(b+2 \beta(d+\mu) I_{0}\right)^{2} \geq \Delta .
\end{array}\right.
$$

We have that (19) is equivalent to

$$
\mathscr{R}_{0} \leq \min \left(p_{1}, p_{2}\right)
$$

According to the discussions above, we can obtain two theorems as follows.

Theorem 1. $E^{*}$ is the unique endemic equilibrium of (1) if and only if $\left(\beta I_{0} / d\right)+1<\mathscr{R}_{0}<p_{0}$.

Note that $p_{1}<p_{2}$ is equivalent to $\beta(d+\mu) I_{0}^{2}<(1-C) B d$. Besides, if $E_{i}$ exist, they must satisfy $\mathscr{R}_{0} \geq p_{0}$ which is equivalent to $\beta(d+\mu) I_{0}^{2}>(1-C) B d$. Therefore, we can obtain that $p_{1}<p_{2}$ is impossible. Note that $p_{1}>p_{2}$ is equivalent to $\beta(d+\mu) I_{0}^{2}>(1-C) B d$. So, we have the following.

Theorem 2. (1) If $\mathscr{R}_{0}<p_{0}$, endemic equilibria $E_{1}$ and $E_{2}$ do not exist.

(2) If $p_{0}<\mathscr{R}_{0}<p_{2}$ and $\beta(d+\mu) I_{0}^{2}>(1-C) B d$, then $E_{1}\left(S_{1}, I_{1}\right)$ and $E_{2}\left(S_{2}, I_{2}\right)$ exist.

(3) If $p_{2}<\mathscr{R}_{0}<p_{1}$ and $\beta(d+\mu) I_{0}^{2}>(1-C) B d$, then $E_{1}\left(S_{1}, I_{1}\right)$ exists.

According to Theorems 1 and 2, the changes in the number of equilibrium with the basic reproduction number are shown in Figure 2 . When $p_{0}<\mathscr{R}_{0}<p_{2}$, there are three endemic equilibria; when $p_{2}<\mathscr{R}_{0}<p_{1}$, two endemic equilibria exist. We can see that the number of infected is proportional to $\mathscr{R}_{0}$ if there is no transport. On the contrary, the number of infected does not always increase with $\mathscr{R}_{0}$ when there is transport. Consequently, the proper transport might reduce the chance of infection in this area.

Next, we give the relationship between the transport rate $B$ and $I$ in Figure 3. As seen from the Figure 3, model (1) has two endemic equilibria when there is transport. When $B<B^{*}$ or $B>B^{*}$ and $I<I^{*}$, the number of the infected always increases with the increase of $B$; when $B>B^{*}$ and $I>I^{*}$, transport is good for disease control. So, the transport has dual effects on the number of $I$; it can not only promote the growth of brucellosis but also inhibit the growth of the disease. Consequently, the transport rate $B$ plays a very important role.

\section{Stability Analysis}

$J$ is the Jacobian matrix of (1) with respect to $E^{*}$ :

$$
J=\left(\begin{array}{cc}
-d \mathscr{R}_{0} & -(d+u) \\
d\left(\mathscr{R}_{0}-1\right) & 0
\end{array}\right),
$$

with $\operatorname{tr} J=-d \mathscr{R}_{0}<0, \quad \operatorname{det} J=d(d+u)\left(\mathscr{R}_{0}-1\right) . \quad$ Then, $\lambda_{1}<0, \lambda_{2}<0$, and we have the following result [22-24].

Theorem 3. $E^{*}$ is locally asymptotically when $\mathscr{R}_{0}>(\beta / d)$ $I_{0}+1$.

This conclusion can be easily obtained. The dynamic behavior of the stable node indicates that susceptible and infected animal populations can coexist for a long time.

The Jacobian matrix of system (1) with respect to $E_{i}$ is

$$
J=\left(\begin{array}{cc}
-d \mathscr{R}_{0} & -(d+u) \\
\beta I & \beta \frac{A-B C}{\beta I+d}-d-\mu
\end{array}\right),
$$

with

$$
\begin{aligned}
\operatorname{det} J & =-\beta(A-B C)+(d+\mu)(\beta I+d)+\frac{\beta^{2} I(A-B C)}{\beta I+d} \\
& =(d+u)(\beta I+d)^{2}<d \beta(A-B C) .
\end{aligned}
$$

If $\operatorname{det} J<0$, then $E_{1}$ is a saddle. Note that $I<I_{0}$, and we have the following results [25-27].

Theorem 4. (1) $E_{1} \quad \begin{aligned} & \text { is } \\ & \left(\beta I_{0}+d\right)^{2}(d+\mu)<d \beta(A-B C) .\end{aligned} \quad$ if

(2) $E_{2}$ is stable if

$$
\left\{\begin{array}{l}
\beta A<\beta B C+\beta^{2} I_{0}^{2}+3 d \beta I_{0}+2 d^{2}+\mu \beta I_{0}+\mu d, \\
(d+\mu)\left(\beta I_{0}+d\right)^{2}+d \beta B C>\max \left\{d(d+\mu)+2 \beta I_{0}(d+\mu)+B \beta,\right. \\
\left.d^{2}+d \mu+\beta d I_{0}+\beta \mu I_{0}+B \beta+\frac{(1-C) B d}{I_{0}}\right\} .
\end{array}\right.
$$




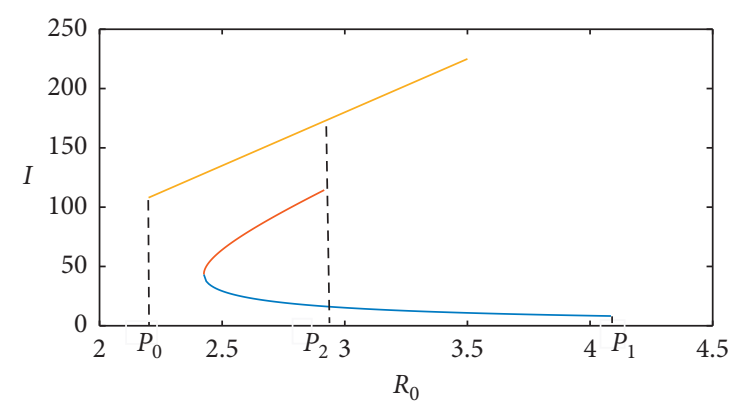

Figure 2: The density of the infected animal populations as a function of basic reproduction number $\mathscr{R}_{0} . P_{0}$ indicates that the equilibrium will appear when there is no transport, $P_{1}$ indicates that the equilibrium will disappear in the case of transport, and $P_{2}$ indicates that there are three equilibria in two cases. Parameter values: $B=50, I_{0}=110, d=0.9, \mu=0.3, \beta=0.01$, and $C=0.5$.

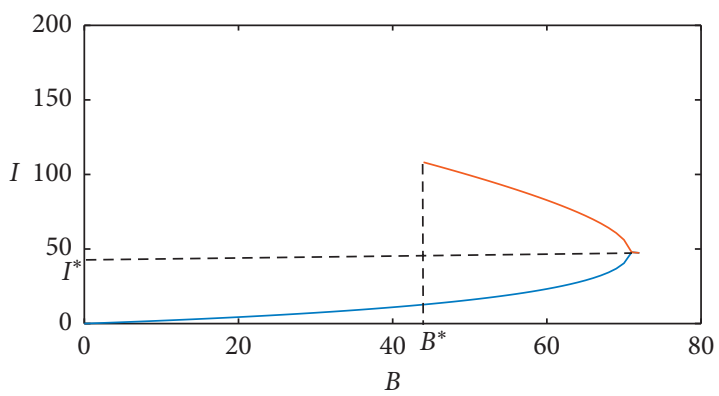

Figure 3: The relationship between the transport rate $B$ and the infected animal populations $I . B^{*}$ indicates that there are two equilibria. Parameter values: $I_{0}=110, d=0.9, \mu=0.3, \beta=0.01, C=0.5$, and $A=300$.

(3) $E_{2}$ is unstable if either

$$
\left\{\begin{array}{l}
\beta A>\beta B C+\beta^{2} I_{0}^{2}+3 d \beta I_{0}+2 d^{2}+\mu \beta I_{0}+\mu d, \\
(d+\mu)\left(\beta I_{0}+d\right)^{2}+d \beta B C>\max \left\{d(d+\mu)+2 \beta I_{0}(d+\mu)+B \beta,\right. \\
\left.d^{2}+d \mu+\beta d I_{0}+\beta \mu I_{0}+B \beta+\frac{(1-C) B d}{I_{0}}\right\},
\end{array}\right.
$$

or

$$
\left\{\begin{array}{l}
(d+\mu)\left(\beta I_{0}+d\right)^{2}+d \beta B C>\max \left\{d(d+\mu)+2 \beta I_{0}(d+\mu)+B \beta,\right. \\
\left.d^{2}+d \mu+\beta d I_{0}+\beta \mu I_{0}+B \beta+\frac{(1-C) B d}{I_{0}}\right\} .
\end{array}\right.
$$

Theorem 5. The unique endemic equilibrium $E^{*}\left(S^{*}, I^{*}\right)$ of system (1) is globally asymptotically stable when $\mathscr{R}_{0}>1+$ $(\beta / d) I_{0}$.

Proof. Define the Lyapunov function [28, 29]:

$$
V(S, I)=S-S^{*}-S^{*} \ln \frac{S}{S_{0}}+I-I^{*}-I^{*} \ln \frac{I}{I_{0}} .
$$

The derivative of $V(S, I)$ along solutions of system (1) is

$$
\begin{aligned}
\frac{\mathrm{d} V}{\mathrm{~d} t} & =\dot{S}\left(1-\frac{S^{*}}{S}\right)+\dot{I}\left(1-\frac{I^{*}}{I}\right) \\
& =(A-\beta S I-d S)\left(1-\frac{S^{*}}{S}\right)+(\beta S I-(d+\mu) I)\left(1-\frac{I^{*}}{I}\right) \\
& =d S^{*}\left\{2-\frac{S}{S^{*}}-\frac{S^{*}}{S}\right\}+\beta S^{*} I^{*}\left\{2-\frac{S}{S^{*}}-\frac{S^{*}}{S}\right\} .
\end{aligned}
$$

Since $\quad\left(S / S^{*}\right)-\left(S^{*} / S\right)<2, \quad(\mathrm{~d} V / \mathrm{d} t)=\left(d S^{*}+\beta S^{*} I^{*}\right)$ $\left(2-\left(S / S^{*}\right)-\left(S^{*} / S\right)\right) \leq 0$.

When $\mathscr{R}_{0}>1+(\beta / d) I_{0}$, we have $(\mathrm{d} V / \mathrm{d} t)<0 .(\mathrm{d} V / \mathrm{d} t)=$ 0 holds if and only if $S=S^{*}, I=I^{*}$. By Lyapunov-Lasalle theorem [30-32], the endemic equilibrium $E^{*}\left(S^{*}, I^{*}\right)$ is globally asymptotically stable when $\mathscr{R}_{0}>1+(\beta / d) I_{0}$.

\section{Numerical Results}

In an epidemic model, the basic reproduction number $\mathscr{R}_{0}$ is an important parameter which can predict the dynamics of the disease. If $\mathscr{R}_{0}<1$, it indicates that the disease can be controlled and can even disappear. Otherwise, the disease will outbreak when the basic reproduction number $\mathscr{R}_{0}>1$. In this paper, when the endemic equilibrium exists, the basic reproduction number $\mathscr{R}_{0}>1$. Next, we analyze the spread of the disease over time.

Figure 4 represents time series of the infected animal populations with different initial values. We take $B=50, I_{0}=110, d=0.9, \mu=0.3, \beta=0.01, c=0.5$, and $A=$ 441.99 with different initial values: (a) $S_{0}=200, I_{0}=130$; (b) $S_{0}=100, I_{0}=80$; (c) $S_{0}=300, I_{0}=180$; (d) $S_{0}=300, I_{0}=$ 100. One can conclude from this figure that there is great distinction between different initial values. As seen from Figure 4(b), the number of infected decreases over time. On the contrary, in the other three figures, the number of infected increases in the beginning. Therefore, initial values play an important role in the control of the disease.

Next, we give sensitivity analysis of $\mathscr{R}_{0}$ in order to find better control strategies. Different parameters are substituted into the expression $\mathscr{R}_{0}=\beta A /(d(d+u))$. We use the normalized forward sensitivity index and derive an analytical expression [33]:

$$
\gamma_{p}^{\mathscr{R}_{0}}=\frac{\partial \mathscr{R}_{0}}{\partial p} \times \frac{\partial p}{\partial \mathscr{R}_{0}} .
$$

Table 1 shows the sensitivity index of $\mathscr{R}_{0}$ with respect to different parameters. We can conclude from Table 1 that $\mathscr{R}_{0}$ is more sensitive to the recruitment rate $A$ and the infection coefficient $\beta$, which means that the main influencing factors of $\mathscr{R}_{0}$ are $A$ and $\beta$. Since $A$ and $\beta$ are proportional to $\mathscr{R}_{0}$, the most effective way to reduce $\mathscr{R}_{0}$ is to reduce the recruitment rate and the infection coefficient. 


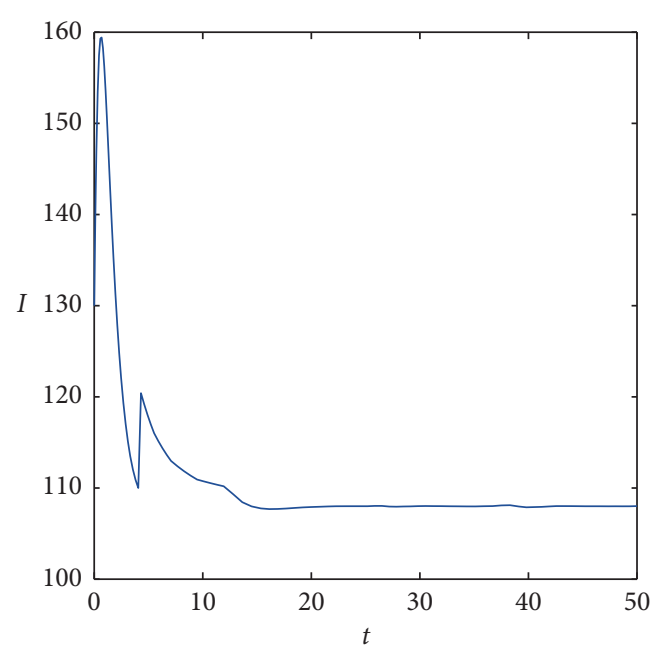

(a)

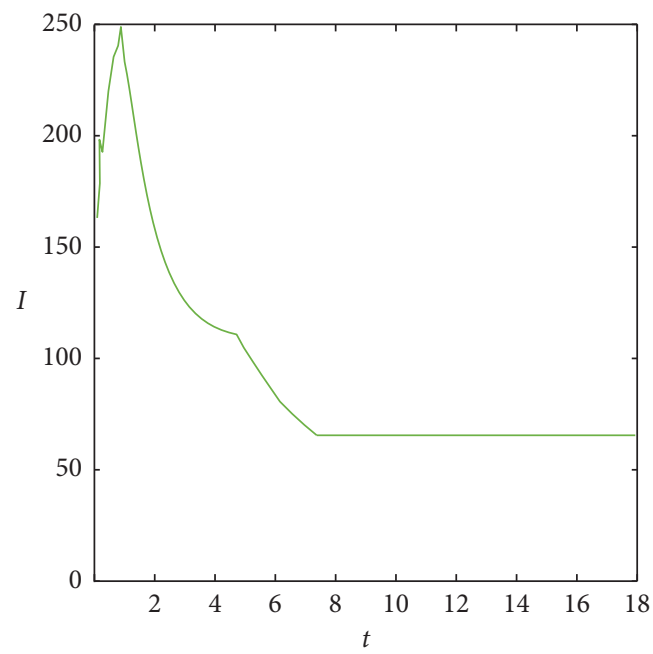

(c)

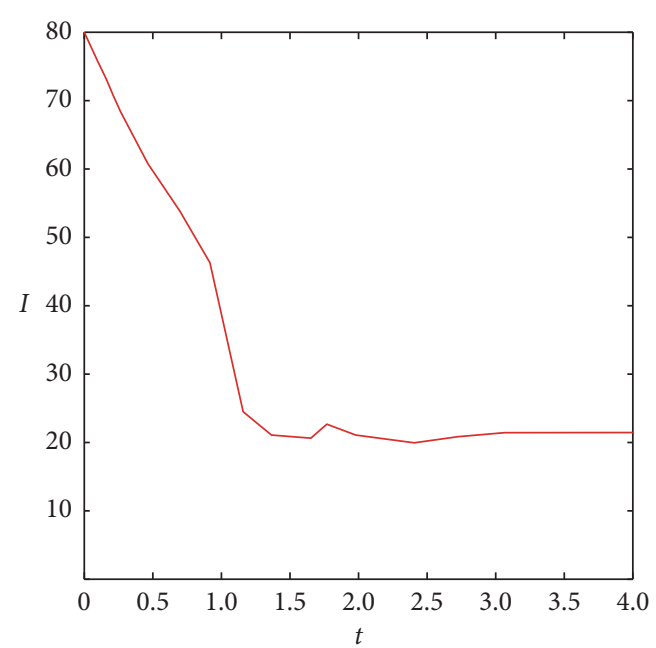

(b)

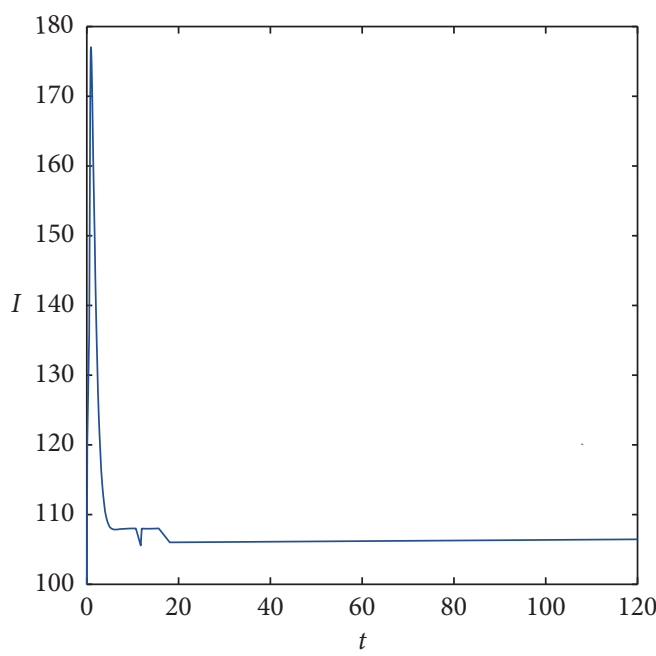

(d)

FIgURE 4: Time series of the infected animal populations with different initial values. Parameter values: $B=50, I_{0}=110, d=0.9, \mu=0.3, \beta=0.01, c=0.5$, and $A=441.99$. Initial values: (a) $S_{0}=200, I_{0}=130 ; \quad$ (b) $S_{0}=100, I_{0}=80 ; \quad$ (c) $S_{0}=300, I_{0}=180 ;$ (d) $S_{0}=300, I_{0}=100$.

TABLE 1: The sensitivity index of $\mathscr{R}_{0}$ with respect to different parameters.

\begin{tabular}{lc}
\hline Parameter & Sensitivity index \\
\hline$\beta$ & 1 \\
$A$ & 1 \\
$\mu$ & -1.836 \\
$d$ & -4.282 \\
\hline
\end{tabular}

\section{Conclusion and Discussion}

Brucellosis has always been a research focus in infectious diseases. Measures to control the spread of brucellosis are vaccinating susceptible and culling infected animal populations. However, in fact, due to some practical factors, such as the incubation period of the disease and economic factor, the breeders will transport the animals containing the infected and fail to take effective measures in time. This case is tremendously overlooked despite its real existence in China. In order to study the influence of transport on the spread of brucellosis, we present an SI model with transport. Through mathematical analysis, we obtain the basic reproduction number $\mathscr{R}_{0}$ and the positive equilibria. The existence and global stability of these equilibria are analyzed. Our results reveal that initial values have important effects on the spread of disease.

Our work demonstrates that the transport has dual effects. Specifically, transport can lead to increase in the number of the infected; besides, transport can also reduce the number of the infected animals in a certain range. This is an interesting result. This indicates that transport can help control the disease for a time. Besides, sensitivity of the basic reproduction number $\mathscr{R}_{0}$ is calculated. The results show that the main influencing factors are the recruitment rate and the infection coefficient. Some measures should be taken, such as vaccination and disinfection. We suggest that breeders 
can increase animal vaccination coverage rate and enhance the awareness of disease prevention.

The analysis shows that the number of infected animals can be controlled if animals are transported reasonably. However, we do not analyze how the disease spreads in the transported animals; this may not be good at disease control. Consequently, we will analyze the dynamic behavior of the animals which have been transported and give a better strategy to control brucellosis. Besides, infectious diseases spread in space, and spatial infectious disease models can describe the dynamics of disease transmission. Therefore, we will integrate the diffusion term into the disease model in the future study [34-36].

\section{Data Availability}

No data were used to support this study.

\section{Conflicts of Interest}

The authors declare that they have no conflicts of interest.

\section{Acknowledgments}

This research was supported by the Natural Science Foundation of Taiyuan Institute of Technology (grant no. 2016LZ02), Program for the (Reserved) Discipline Leaders of Taiyuan Institute of Technology (no. 2018008), and Natural Science Foundation of Shanxi Province (no. 201901D111322).

\section{References}

[1] J. Zinsstag, F. Roth, D. Orkhon et al., "A model of animalhuman brucellosis transmission in Mongolia," Preventive Veterinary Medicine, vol. 69, no. 1-2, pp. 77-95, 2005.

[2] J. Gonzalez-Guzman and R. Naulin, "Analysis of a model of bovine brucellosis using singular perturbations," Journal of Mathematical Biology, vol. 33, no. 2, pp. 211-223, 1994.

[3] G. Pappas, N. Akritidis, M. Bosilkovski, and E. Tsianos, "Brucellosis and the respiratory system," Clinical Infectious Diseases, vol. 37, no. 7, pp. 95-99, 2003.

[4] M. Doganay and B. Aygen, "Human brucellosis: an overview," International Journal of Infectious Diseases, vol. 7, no. 3, pp. 173-182, 2003.

[5] R. S. Cantrell, C. Cosner, and W. F. Faganantrell, "Brucellosis, botflies, and brainworms: the impact of edge habitats on pathogen transmission and species extinction," Journal of Mathematical Biology, vol. 42, no. 2, pp. 95-119, 2001.

[6] M.-T. Li, G.-Q. Sun, Y.-F. Wu, J. Zhang, and Z. Jin, "Transmission dynamics of a multi-group brucellosis model with mixed cross infection in public farm," Applied Mathematics and Computation, vol. 237, pp. 582-594, 2014.

[7] B. Ruben, J. D. Band, P. Wong, and J. Colville, "Person-toperson transmission of Brucella melitensis," Lancet, vol. 337, no. 8732, pp. 14-15, 1982.

[8] G.-Q. Sun, C.-H. Wang, and Z.-Y. Wu, "Pattern dynamics of a Gierer-Meinhardt model with spatial effects," Nonlinear Dynamics, vol. 88, no. 2, pp. 1385-1396, 2017.

[9] Q. Hou, X. Sun, Y. Wang, B. Huang, and Z. Jin, "Global properties of a general dynamic model for animal diseases: a case study of brucellosis and tuberculosis transmission,"
Journal of Mathematical Analysis and Applications, vol. 414, no. 1, pp. 424-433, 2014.

[10] Q. Hou, X. Sun, J. Zhang, Y. Liu, Y. Wang, and Z. Jin, "Modeling the transmission dynamics of sheep brucellosis in inner Mongolia autonomous region, China," Mathematical Biosciences, vol. 242, no. 1, pp. 51-58, 2013.

[11] G.-Q. Sun and Z.-K. Zhang, "Global stability for a sheep brucellosis model with immigration," Applied Mathematics and Computation, vol. 246, pp. 336-345, 2014.

[12] N. Jing and G. Q. Sun, "Modeling the transmission dynamics of dairy cattle brucellosis in Jilin province, China," Journal of Biological System, vol. 22, no. 04, pp. 533-554, 2014.

[13] J. Yang, R. Xu, and J. Li, "Threshold dynamics of an age-space structured brucellosis disease model with Neumann boundary condition," Nonlinear Analysis: Real World Applications, vol. 50, pp. 192-217, 2019.

[14] C. Y. Yang, P. O. Lolika, S. Mushayabasa, and J. Wang, "Modeling the spatiotemporal variations in brucellosis transmission," Nonlinear Analysis: Real World Applications, vol. 38, pp. 49-67, 2017.

[15] B. Aïnseba, C. Benosman, and P. Magal, "A model for ovine brucellosis incorporating direct and indirect transmission," Journal of Biological Dynamics, vol. 4, no. 1, pp. 2-11, 2010.

[16] J. Liu, J. Wu, and Y. Zhou, "Modeling disease spread via transport-related infection by a delay differential equation," Rocky Mountain Journal of Mathematics, vol. 38, no. 5, pp. 1525-1540, 2008.

[17] X. Liu and Y. Takeuchi, "Spread of disease with transportrelated infection and entry screening," Journal of Theoretical Biology, vol. 242, no. 2, pp. 517-528, 2006.

[18] Y. Takeuchi, X. Liu, and J. Cui, "Global dynamics of SIS models with transport-related infection," Journal of Mathematical Analysis and Applications, vol. 329, no. 2, pp. 14601471, 2007.

[19] J. Liu, Z. Bai, and T. Zhang, "A periodic two-patch SIS model with time delay and transport-related infection," Journal of Theoretical Biology, vol. 437, pp. 36-44, 2018.

[20] X. Liu and P. Stechlinski, "Transmission dynamics of a switched multi-city model with transport-related infections," Nonlinear Analysis: Real World Applications, vol. 14, no. 1, pp. 264-279, 2013.

[21] O. Diekmann, J. A. P. Heesterbeek, and J. A. J. Metz, "On the definition and the computation of the basic reproduction ratio $\mathscr{R}_{0}$ in models for infectious diseases in heterogeneous populations," Journal of Mathematical Biology, vol. 28, no. 4, pp. 365-382, 1990.

[22] A. Q. Khan, "Stability and Neimark-Sacker bifurcation of a ratio-dependence predator-prey model," Mathematical Methods in the Applied Sciences, vol. 40, pp. 404109-404117, 2017.

[23] G.-Q. Sun, C.-H. Wang, L.-L. Chang, Y.-P. Wu, L. Li, and Z. Jin, "Effects of feedback regulation on vegetation patterns in semi-arid environments," Applied Mathematical Modelling, vol. 61, pp. 200-215, 2018.

[24] L. Li, J. Zhen, and S. Gui-Quan, "Spatial pattern of an epidemic model with cross-diffusion," Chinese Physics Letters, vol. 25, no. 9, pp. 3500-3503, 2008.

[25] G.-Q. Sun, J.-H. Xie, S.-H. Huang, Z. Jin, M.-T. Li, and L. Liu, "Transmission dynamics of cholera: mathematical modeling and control strategies," Communications in Nonlinear Science and Numerical Simulation, vol. 45, pp. 235-244, 2017.

[26] L. Li, G.-Q. Sun, and Z. Jin, "Bifurcation and chaos in an epidemic model with nonlinear incidence rates," Applied 
Mathematics and Computation, vol. 216, no. 4, pp. 1226-1234, 2010.

[27] G.-Q. Sun, "Pattern formation of an epidemic model with diffusion," Nonlinear Dynamics, vol. 69, no. 3, pp. 1097-1104, 2012.

[28] X. Zhang and X. Liu, "Backward bifurcation of an epidemic model with saturated treatment function," Journal of Mathematical Analysis and Applications, vol. 348, no. 1, pp. 433443, 2008.

[29] W. D. Wang, "Backward bifurcation of an epidemic model with treatment," Mathematical Biosciences, vol. 201, no. 1-2, pp. 58-71, 2006.

[30] J. Hespanha, "Uniform stability of switched linear systems: extensions of LaSalle's invariance principle," IEEE Transactions on Automatic Control, vol. 49, no. 4, pp. 470-482, 2004.

[31] J. P. LaSalle, "Stability theory for ordinary differential equations," Journal of Differential Equations, vol. 4, no. 1, pp. 57-65, 1968.

[32] J. P. LaSalle, "Stability theory and invariance principles," Dynamical Systems, vol. 1, pp. 211-222, 1976.

[33] N. Chitnis, J. M. Hyman, and J. M. Cushing, "Determining important parameters in the spread of malaria through the sensitivity analysis of a mathematical model," Bulletin of Mathematical Biology, vol. 70, no. 5, pp. 1272-1296, 2008.

[34] G.-Q. Sun, M. Jusup, Z. Jin, Y. Wang, and Z. Wang, "Pattern transitions in spatial epidemics: mechanisms and emergent properties," Physics of Life Reviews, vol. 19, pp. 43-73, 2016.

[35] Z. G. Guo, G. Q. Sun, Z. Wang et al., "Spatial dynamics of an epidemic model with nonlocal infection," Applied Mathematics and Computation, vol. 377, Article ID 125158, 2020.

[36] Z. G. Guo, L. P. Song, G. Q. Sun, C. Li, and Z. Jin, "Pattern dynamics of an SIS epidemic model with nonlocal delay," International Journal of Bifurcation and Chaos, vol. 29, no. 2, Article ID 1950027, 2019. 